\title{
An Efficient Algorithm for Multimodal Medical Image Fusion based On Feature Selection and PCA Using DTCWT
}

\author{
Type of article: Conference abstract \\ Abdallah bengueddoudj ${ }^{1}$, Zoubeida Messali ${ }^{1}$ \\ 1. Department of Electrical Engineering, University of Bordj Bou Arreridj, Algeria \\ Beng.abdallah@hotmail.com
}

\begin{abstract}
:
Background: In the two past decades, medical image fusion has become an essential part of modern medicine due to the availability of numerous imaging modalities (MRI, CT, SPECT. etc). This paper presents a new medical image fusion algorithm based on DTCWT and uses different fusion rules in order to obtain a new image which contains more information than any of the input images.

Methods: In order to improve the visual quality of the fused image, we propose a new image fusion algorithm based on Dual Tree Complex Wavelet Transform (DTCWT). Using different fusion rules in a single algorithm leads to a perfect reconstruction of the output (fused image).This combination will create a new method which exploits the advantages of each method separately. DTCWT present good directionality since it considers the edge information in six directions and provides approximate shift invariant. The goal of Principal Component Analysis (PCA) is to extract the most significant features (wavelet coefficients in our case) in order to improve the spatial resolution. The proposed algorithm fuses the detailed wavelet coefficients of input images using features selection rule.

Results: We have conducted several experiments over different sets of multimodal medical images such as CT/MRI, MRA/T1-MRI; however, only results of two sets have been presented (due to pages-limit). The proposed fusion algorithm is compared to recent fusion methods presented in the literature (eight methods) in terms of visual quality and quantitatively using well known fusion performance metrics (five metrics). Results showed that the proposed algorithm outperforms the existing ones in terms of visual and quantitative evaluations.

Conclusion: This paper focuses on image fusion of medical images obtained from different modalities. We have proposed a novel algorithm based on DTCWT in order to merge multimodal medical images. Experiments have been performed over two different sets of multimodal medical images. The results show that the proposed method significantly outperforms other techniques reported in the literature.
\end{abstract}

Keywords: Multimodal medical images; Image fusion, DTCWT, PCA, Feature selection

\section{Declaration of conflicts}

This article is selected from the abstract's book of the International Conference on Health Sciences and Medical Technologies,10-12 October 2017, Tlemcen, Algeria, ICHSMT'17.

\section{Authors' biography}

No biography 
Medical Technologies Journal, Volume: 1, Issue: 3, July -September 2017, Pages: 60-61. DOI: https://doi.org/10.26415/2572-004X-vol1iss3p60-61

\section{References}

No references 